\title{
A pilot study of fluorescence lifetime imaging ophthalmoscopy in preclinical Alzheimer's disease
}

\author{
SriniVas R. Sadda ${ }^{1,2} \cdot$ Enrico Borrelli ${ }^{1}{ }^{1} \cdot$ Wenying Fan ${ }^{1} \cdot$ Adel Ebraheem $^{1} \cdot$ Kenneth M. Marion $\mathbb{D}^{1} \cdot$ \\ Michael Harrington ${ }^{3} \cdot$ Soonil Kwon ${ }^{1,4}$
}

Received: 18 December 2018 / Revised: 6 February 2019 / Accepted: 24 February 2019 / Published online: 28 March 2019

(c) The Royal College of Ophthalmologists 2019

\begin{abstract}
Purpose To investigate fluorescence lifetime imaging ophthalmoscopy (FLIO) findings in preclinical Alzheimer's disease $(\mathrm{AD})$.

Methods This prospective, observational study enrolled patients with early AD undergoing Alzheimer's biomarker analysis and matched controls. Alzheimer-associated parameters ( $\beta$-amyloid [A $\beta]$, total tau in cerebrospinal fluid [CSF], Mini-Mental Status Examination [MMSE], etc.), risk factor-associated data (body mass index [BMI], hypertension, lipid profile, etc.), ganglion cell layer plus inner plexiform layer (GCIPL) thickness in structural optical coherence tomography (OCT), OCT angiography data, and FLIO-derived parameters $\left(\tau_{\mathrm{m}}, \tau_{1}, \tau_{2}\right.$, and $\left.\tau_{3}\right)$ in short and long spectral channels (SSC and LSC) were compared and correlated between the two groups. Additional analyses were performed separately within subgroups of phakic and pseudophakic.

Results A total of 28 eyes from 15 subjects ( 8 control and 7 AD) were included in this analysis. In FLIO parameters, $\tau_{\mathrm{m}}$ in $\mathrm{AD}$ group showed longer lifetimes compared to the controls in phakic subjects $(593.9 \pm 93.3,454.4 \pm 38.6 \mathrm{ps} ; 475.0 \pm 71.6$, $394.1 \pm 28.2 \mathrm{ps}$ in SSC and LSC of AD and control groups, respectively, $p=0.036$ and 0.024$)$. A $\beta$, tau in CSF, and GCIPL thickness correlated with $\tau_{\mathrm{m}}$ in the LSC for phakic subjects $(r=-0.611$ to $0.562, p<0.05$ for all), but only the GCIPL thickness showed a correlation with FLIO parameters in pseudophakic subjects ( $r=-0.893$ to $-0.795, p<0.001$ for all). Conclusion FLIO-derived parameters appear to correlate with A $\beta$, tau levels in the CSF, and GCIPL thickness on OCT in $\mathrm{AD}$ patients. If these findings can be validated in future longitudinal studies, FLIO may prove to be useful as a simple, noninvasive diagnostic tool for $\mathrm{AD}$.
\end{abstract}

Presented at the Annual meeting of the Association for Research in Vision and Ophthalmology, Honolulu, Hawaii, United States, May 3, 2018.

$\triangle$ Soonil Kwon

magicham@hallym.or.kr

1 Doheny Eye Institute, University of California, Los Angeles, CA, USA

2 Department of Ophthalmology, David Geffen School of Medicine at UCLA, Los Angeles, CA, USA

3 Molecular Neurology Program, Huntington Medical Research Institutes, Pasadena, CA, USA

4 Department of Ophthalmology, Hallym Sacred Heart Hospital, Hallym University College of Medicine, Anyang, Gyeonggi, South Korea

\section{Introduction}

Alzheimer's disease (AD) is a chronic, progressive neurodegenerative disease that may result in severe cognitive impairment. Alzheimer's-type dementia is the most common cause of dementia (60-70\%) and its prevalence has been increasing with the lengthening of life expectancies. Globally, there are almost 46 million people living with dementia, with the number expected to rise to 131.5 million by the year 2050 [1]. Abnormally high levels of cerebral $\beta$-amyloid $(\mathrm{A} \beta)$ are indicative of $\mathrm{AD}$ in individuals with clinically classified dementia or mild cognitive impairment (MCI) [2, 3]. However, AD manifests years or decades before the clinical diagnosis of dementia. Recently, therapeutic interventions designed to slow down the aggregation of $A \beta$ have been recommended to patients deemed to present with signs of preclinical AD, preceding MCI, or confirmed AD. Such 
interventions are targeted to help patients in very early stages of the disease [4].

The retina is a part of the central nervous system that shares many similarities with the brain. Several technologies offer the ability to perform non-invasive and highresolution imaging of the retina. There is growing evidence that the presence of $\mathrm{A} \beta$ plaque in the retinas of $\mathrm{AD}$ patients may reflect similar derangements occurring in their brains. It has also been shown that the presence of $A \beta$ plaque in patients with $\mathrm{AD}$ is correlated with retinal structural deficits such as retinal ganglion cell loss, nerve fiber layer atrophy, and thinning of the macular ganglion cell complex [5-8].

Fluorescence lifetime imaging ophthalmoscopy (FLIO) is a novel technique for assessing fluorophore lifetimes, offering the possibility of in vivo molecular contrast [9-11]. The technology applies a laser pulse to excite the retina, resulting in autofluorescence of certain fluorophores. The FLIO device then measures the fluorescence lifetime of these excited fluorophores, derived based on the amount of time it takes for the excited fluorophore to return to its ground state from its higher energy level while releasing a long-wavelength photon. As each fluorophore possesses a characteristic fluorescence lifetime, the decay histogram of the fluorescence intensity after pulsed excitation allows for differentiation of fluorophores with overlapping emission spectra. It can produce quantitative images based on the lifetimes of the different endogenous retinal fluorophores [11-13]. Thus, fluorescence lifetime measurements offer rich amounts of information that may allow for detection of retinal abnormalities at an early stage. It has been reported that FLIO measurements are indicative of retinal changes of AD patients in relation to Alzheimer-specific markers [14]. However, the relationship between FLIO measurements of the retina and preclinical $\mathrm{AD}$ and/or early-stage $\mathrm{AD}$ patients has not yet been explored.

Therefore, the purpose of this study was to investigate the change of FLIO in early-stage Alzheimer disease patients.

\section{Methods}

\section{Subjects}

In this prospective, observational study, subjects with an early-stage of $\mathrm{AD}$ and normal controls who were participating in an ongoing Alzheimer's biomarkers analysis at Huntington Medical Research Institute (HMRI) were included.

These subjects were monitored at HMRI, a specialized dementia center. Brain magnetic resonance imaging (MRI) and cerebrospinal fluid (CSF) investigation for tau protein, and $\mathrm{A} \beta$ were performed in all subjects. Subjects also underwent neuropsychiatric testing, which included Mini-
Mental Status Examination (MMSE), Montreal cognitive assessment (MoCA), global deterioration scale testing, and clinical dementia rating.

Each subject's AD stage was determined according to the cognitive status and the CSF investigation for tau protein and $\mathrm{A} \beta$. The $\mathrm{AD}$ stage of the participants at HMRI could be classified into five different categories: (1) cognitively healthy with normal amyloid/tau, (2) cognitively healthy with Alzheimer-like amyloid/tau, (3) cognitively impaired but not with MCI and/or Alzheimer-like amyloid/ tau, (4) MCI with normal amyloid/tau, and (5) AD with normal amyloid/tau. Subjects who were classified as cognitively healthy, or had slightly impaired cognition (category 1,2 , and 3 ), were included in this study, while subjects with advanced AD or MCI were excluded. Subjects had no ophthalmic disease except mild cataract or pseudophakia as confirmed by medical history and ophthalmic examination. Written informed consent was obtained from all individuals prior to study participation. The study was approved by the Institutional Review Board of the University of California - Los Angeles and conducted in accordance with the ethical standards stated in the Declaration of Helsinki, as well as in compliance with the regulations set forth by the Health Insurance Portability and Accountability Act.

\section{Study design}

All subjects underwent detailed clinical examination, including best-corrected visual acuity, intraocular pressure measurement, slit-lamp biomicroscopy, autorefraction using an ARK-530 (Nidek Co. Ltd., Gamagori, Japan), ultrawidefield fundus photography using an Optos 200Tx (Optos, Dunfermline, UK), and optical coherence tomography (OCT) using a Cirrus HD-OCT model 5000 with Angioplex ${ }^{\mathrm{TM}}$ (Carl Zeiss Meditec, Dublin, CA, USA).

All subjects underwent FLIO measurements in both eyes after dilation. The pupil was dilated to at least $7 \mathrm{~mm}$ in diameter using tropicamide $0.5 \%$ and phenylephrine $\mathrm{HCl}$ $2.5 \%$. All FLIO measurements were acquired by one trained operator (SK).

\section{Setting for FLIO}

Basic principles of the FLIO technique have been described in several reports $[11-13,15]$. A prototype FLIO device was used for acquisition of fluorescence lifetime measurements in the retina (Heidelberg Engineering, Heidelberg, Germany). A $473 \mathrm{~nm}$ pulsed blue laser light at an $80 \mathrm{MHz}$ repetition rate was used for excitation of retinal fluorescence. The fluorescence light was detected by highly sensitive hybrid detectors in two different spectral channels (short spectral channel or SSC: $498-560 \mathrm{~nm}$; long 
spectral channel or LSC: $560-720 \mathrm{~nm}$ ) and registered by time-correlated single photon counting cards. Autofluorescence images were detected simultaneously with a $30^{\circ}$ confocal high-contrast infrared image $(256 \times 256$ pixels), which was used for adjusting spatial location by the system to register each returning photon to the corresponding image pixel. The macula was centrally adjusted in the FLIO exam, and at least 1000 photons were collected per pixel for an adequate signal-to-noise ratio.

\section{Data analysis}

Fluorescence lifetime data were analyzed by the software SPCImage 4.4.2 (Becker\&Hickl GmbH, Berlin, Germany) using a tri-exponential decay model and $3 \times 3$ pixel binning in both channels (Fig. 1), which was same setting applied in our previous studies. For a global characterization of the fluorescence, the mean fluorescence lifetime $\tau_{\mathrm{m}}$ was calculated from the lifetime components $\tau_{1}, \tau_{2}$, and $\tau_{3}$ and their respective amplitudes $a_{1}, a_{2}$, and $a_{3}$ with the equation as follows:

$\tau_{\mathrm{m}}=\left(\alpha_{1} * \tau_{1}+\alpha_{2} * \tau_{2}+\alpha_{3} * \tau_{3}\right) /\left(\alpha_{1}+\alpha_{2}+\alpha_{3}\right)$.
The "goodness" of the exponential fit was assessed by the $\chi^{2}$ value. This application of $\chi^{2}$ has been described in several previous reports [11-13]. Briefly, a value of $\chi^{2}$ close to 1 indicates a good fit for an appropriate model.

Vascular density (VD) was evaluated from the Cirrus HD-OCT. OCTA data was evaluated due to recent reports suggesting retinal vascular alterations in early Alzheimer's disease [15-17]. OCT angiography images $\left(3 \times 3 \mathrm{~mm}^{2}\right)$ using Food Drug Administration-cleared OCTA software (Cirrus Angioplex ${ }^{\mathrm{TM}}$ software version 6.0) were obtained by the Cirrus 5000 with Angioplex ${ }^{\mathrm{TM}}$, which were utilized for the analysis of VD in the superficial and deep vascular plexuses. Our previously reported semiautomated algorithm was used to quantify VD [18, 19].

VD was calculated by dividing the area occupied by vessels (in pixels) by the total area (in pixels). Briefly, the $2 \mathrm{D}$ en face $3 \times 3 \mathrm{~mm}^{2}$ fovea-centered OCTA image of the scanned retina was first converted to an 8-bit image (524x 524 pixels). Then, using Image $J$, the image was converted into a binary image by a three-step method. First, the image was processed using a top-hat filter with a window size of 12 pixels to remove uneven illumination. Global and local
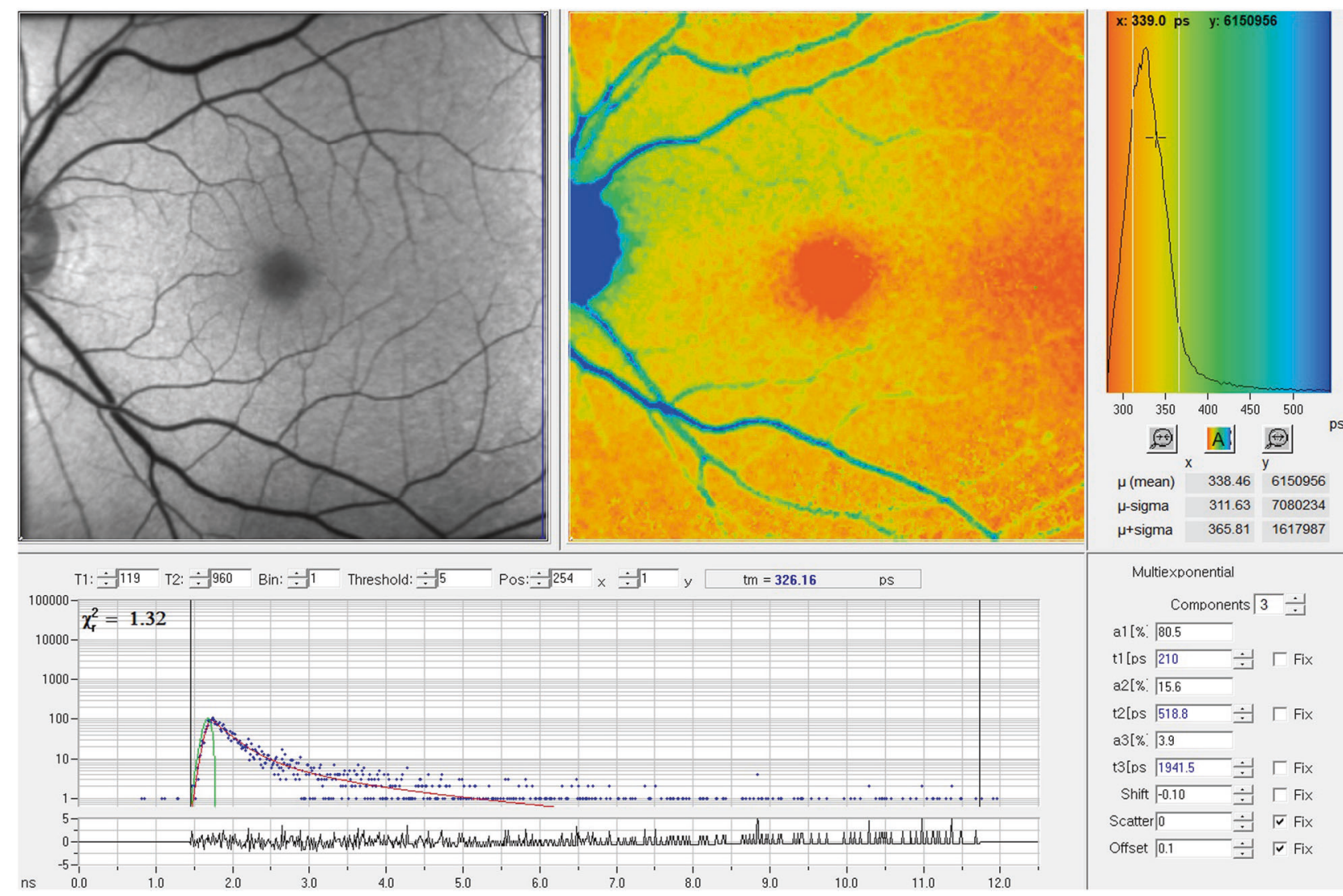

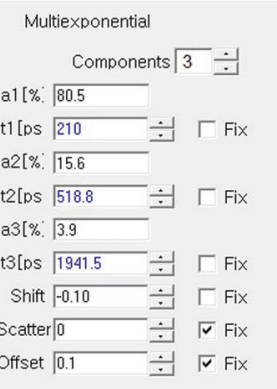

Fig. 1 Representative fluorescence lifetime image ( $\tau_{\mathrm{m}}$ in short spectral channel (SSC)) of an 84-year-old female subject with preclinical Alzheimer's disease 
thresholding (median method) were used separately to create two binary images. Finally, two binary images were combined to create the final binarized version by including the pixels detected by both binary methods. Ganglion cell layer plus inner plexiform layer (GCIPL) thickness from a 3 $\times 3 \mathrm{~mm}^{2}$ area centered on the fovea was obtained automatically from the Cirrus OCT.

\section{Statistical analysis}

Statistical analysis of the data was performed using SPSS 23.0 software (IBM Corporation, NY, USA).

Demographic features, Alzheimer-associated data, risk factor-associated data, and OCT data were investigated with Mann-Whitney $U$ tests to compare the Alzheimer group with normal controls. Fluorescence lifetime parameters were also compared between the two groups. Associations between FLIO parameters $\left(\tau_{\mathrm{m}}, \tau_{1}, \tau_{2}\right.$, and $\left.\tau_{3}\right)$ and other laboratory and diagnostic data from the subjects were assessed using Spearman's correlation analysis. All FLIO analyses were performed separately within subgroups of phakic and pseudophakic as previously suggested due to the known substantial influence of the crystalline lens on fluorescence lifetime values [13]. Statistical results were expressed as $P$ values and a $P$ value of $<0.05$ was considered statistically significant.

\section{Results}

\section{Comparison of clincal data between Alzheimer group and normal controls}

A total of 28 eyes from 15 subjects were included in this analysis. Seven subjects who were considered to be cognitively healthy with Alzheimer-like amyloid or tau level (category 2) or cognitively impaired but not with MCI and/ or Alzheimer-like amyloid/tau (category 3) were assigned to the Alzheimer group and eight subjects who were considered to be cognitively healthy with normal laboratory findings (category 1) were assigned to the normal control group. The mean age was $82.4 \pm 6.8$ in the Alzheimer group and $76.3 \pm 11.9$ in the Normal control group. There was no statistically significant difference in age, sex, and refractive error between the two groups (Table 1). The Alzheimerassociated clinical biomarkers, $A \beta$ and total tau level in the CSF, were statistically different between the two groups (A $\beta$ : $P=0.022$; total tau in CSF: $P=0.005$, Table 1 ). The risk factors for Alzheimer's disease, which include BMI, hypertension, and dyslipidemia, were not statistically significant between the two groups. The OCTA VD results did not show statistically significant differences in the superficial vascular plexus, deep vascular plexus, or the
Table 1 Demographics and clinical data of Alzheimer group and control

\begin{tabular}{|c|c|c|c|}
\hline & Alzheimer & Control & $P$ value \\
\hline Number of subjects (eye) & $7(13)$ & $8(15)$ & \\
\hline Age (years) & $82.4 \pm 6.8$ & $76.3 \pm 11.9$ & 0.189 \\
\hline $\operatorname{Sex}(M: F)$ & $4: 3$ & $3: 5$ & \\
\hline $\begin{array}{l}\text { Lens status (phakic: } \\
\text { pseudophakic) }\end{array}$ & $4: 9$ & $10: 5$ & \\
\hline \multicolumn{4}{|l|}{$\begin{array}{l}\text { Spherical equivalent, } \\
\text { mean } \pm \text { SD (D) }\end{array}$} \\
\hline Right eye & $0.4 \pm 3.9$ & $-0.7 \pm 2.2$ & 0.423 \\
\hline Left eye & $0.5 \pm 3.8$ & $-0.5 \pm 2.1$ & \\
\hline \multicolumn{4}{|l|}{ Alzheimer-associated data } \\
\hline$\beta$-Amyloid in CSF (pg/ml) & $523 \pm 136$ & $780 \pm 211$ & $0.022^{\mathrm{a}}$ \\
\hline Total tau of CSF (pg/ml) & $630 \pm 319$ & $170 \pm 46$ & $0.005^{\mathrm{a}}$ \\
\hline MMSE-7 & $29 \pm 0.8$ & $30 \pm 0.8$ & 0.128 \\
\hline $\mathrm{MoCA}$ & $26 \pm 1.1$ & $26 \pm 1.7$ & 0.902 \\
\hline \multicolumn{4}{|l|}{ Risk factor-associated data } \\
\hline Hypertension & 2 & 4 & \\
\hline Diabetes & 0 & 0 & \\
\hline Dyslipidemia & 3 & 1 & \\
\hline BMI & $26 \pm 6.3$ & $26 \pm 6.3$ & 0.902 \\
\hline BUN & $18 \pm 3.6$ & $19 \pm 3.9$ & 0.902 \\
\hline CRP & $3 \pm 5.0$ & $2 \pm 1.5$ & 0.836 \\
\hline Total cholesterol & $184 \pm 50$ & $189 \pm 42$ & 0.902 \\
\hline Homocysteine & $12 \pm 1.5$ & $11 \pm 2.5$ & 0.589 \\
\hline \multicolumn{4}{|l|}{ OCT data } \\
\hline GCIPL thickness & $66 \pm 2.3$ & $73 \pm 5.3$ & $0.002^{\mathrm{a}}$ \\
\hline Superficial VD & $38 \pm 1.8$ & $37 \pm 0.9$ & 0.266 \\
\hline Deep VD & $31 \pm 2.0$ & $32 \pm 1.7$ & 0.101 \\
\hline Choriocapillary VD & $88 \pm 3.7$ & $89 \pm 2.6$ & 0.671 \\
\hline
\end{tabular}

CSF cerebrospinal fluid, MMSE Mini-Mental State Examination, MoCA Montreal Cognitive Assessment, BMI body mass index, BUN blood urea nitrogen, $C R P$ C-reactive protein, GCIPL ganglion cell layer + inner plexiform layer, $V D$ vascular density

Reference value: $\beta$-Amyloid in CSF $>500 \mathrm{pg} / \mathrm{ml}$; total tau in CSF $<450 \mathrm{pg} / \mathrm{ml}$

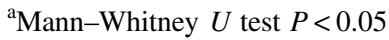

choriocapillaris between the two groups. However, the GCIPL thickness measured using structural OCT was significantly decreased in the Alzheimer group ( $66 \pm 2.3$ vs. 73 $\pm 5.3 \mu \mathrm{m}, P=0.002$, Table 1$)$.

\section{Comparison of fluoresence lifetime between Alzheimer group and normal controls}

The average mean fluorescence lifetime $\left(\tau_{\mathrm{m}}\right)$ of the entire scan area was $469.5 \pm 133.6 \mathrm{ps}$ in SSC and $421.7 \pm 42.1 \mathrm{ps}$ in the LSC in the Alzheimer group, while the control group averaged $437.7 \pm 80.3 \mathrm{ps}$ and $402.5 \pm 41.0 \mathrm{ps}$ in the SSC and LSC, respectively (Table 2). There was no statistically 
Table 2 Fluorescence lifetime of Alzheimer group and control

\begin{tabular}{|c|c|c|c|}
\hline & Alzheimer & Control & $P$ value \\
\hline \multicolumn{4}{|l|}{ All subjects } \\
\hline Number (eyes) & 13 & 15 & \\
\hline \multicolumn{4}{|l|}{$\tau_{\mathrm{m}}(\mathrm{ps})$} \\
\hline SSC & $469.5 \pm 133.6$ & $437.7 \pm 80.3$ & 0.786 \\
\hline LSC & $421.7 \pm 42.1$ & $402.5 \pm 41.0$ & 0.185 \\
\hline \multicolumn{4}{|l|}{$\tau_{1}(\mathrm{ps})$} \\
\hline $\mathrm{SSC}$ & $229.6 \pm 30.7$ & $221.6 \pm 17.9$ & 1.000 \\
\hline LSC & $242.8 \pm 15.9$ & $233.8 \pm 18.9$ & 0.486 \\
\hline \multicolumn{4}{|l|}{$\tau_{2}(\mathrm{ps})$} \\
\hline $\mathrm{SSC}$ & $1057.7 \pm 347.8$ & $1029.4 \pm 296.6$ & 0.821 \\
\hline LSC & $931.6 \pm 75.1$ & $910.0 \pm 73.1$ & 0.650 \\
\hline \multicolumn{4}{|l|}{$\tau_{3}(\mathrm{ps})$} \\
\hline SSC & $5266.4 \pm 2194.3$ & $5167.9 \pm 1989.9$ & 0.751 \\
\hline LSC & $3427.7 \pm 590.1$ & $3323.2 \pm 467.1$ & 0.717 \\
\hline \multicolumn{4}{|c|}{ Pseudophakic subjects } \\
\hline Number (eyes) & 9 & 5 & \\
\hline \multicolumn{4}{|l|}{$\tau_{\mathrm{m}}(\mathrm{ps})$} \\
\hline SSC & $414.3 \pm 130.3$ & $363.0 \pm 29.0$ & 0.699 \\
\hline LSC & $407.2 \pm 27.2$ & $419.4 \pm 79.8$ & 0.898 \\
\hline \multicolumn{4}{|l|}{$\tau_{1}(\mathrm{ps})$} \\
\hline SSC & $215.4 \pm 25.9$ & $207.0 \pm 21.5$ & 0.797 \\
\hline LSC & $239.3 \pm 11.4$ & $240.9 \pm 36.8$ & 0.797 \\
\hline \multicolumn{4}{|l|}{$\tau_{2}(\mathrm{ps})$} \\
\hline SSC & $913.2 \pm 376.4$ & $750.1 \pm 49.9$ & 0.797 \\
\hline LSC & $899.2 \pm 63.1$ & $869.0 \pm 144.8$ & 1.000 \\
\hline \multicolumn{4}{|l|}{$\tau_{3}(\mathrm{ps})$} \\
\hline SSC & $4379.4 \pm 2339.5$ & $3275.1 \pm 314.9$ & 0.797 \\
\hline LSC & $3169.3 \pm 617.3$ & $2860.8 \pm 288.2$ & 0.699 \\
\hline \multicolumn{4}{|l|}{ Phakic subjects } \\
\hline Number (eyes) & 4 & 10 & \\
\hline \multicolumn{4}{|l|}{$\tau_{\mathrm{m}}(\mathrm{ps})$} \\
\hline SSC & $593.9 \pm 93.3$ & $475.0 \pm 71.6$ & $0.036^{\mathrm{a}}$ \\
\hline LSC & $454.4 \pm 38.6$ & $394.1 \pm 28.2$ & $0.024^{\mathrm{a}}$ \\
\hline \multicolumn{4}{|l|}{$\tau_{1}(\mathrm{ps})$} \\
\hline SSC & $261.4 \pm 9.8$ & $229.0 \pm 15.0$ & $0.008^{\mathrm{a}}$ \\
\hline LSC & $250.8 \pm 15.8$ & $230.2 \pm 13.2$ & 0.054 \\
\hline \multicolumn{4}{|l|}{$\tau_{2}(\mathrm{ps})$} \\
\hline SSC & $1382.7 \pm 113.7$ & $1169.1 \pm 267.4$ & 0.076 \\
\hline LSC & $1004.5 \pm 39.9$ & $930.5 \pm 43.7$ & $0.014^{\mathrm{a}}$ \\
\hline \multicolumn{4}{|l|}{$\tau_{3}(\mathrm{ps})$} \\
\hline SSC & $7262.0 \pm 624.0$ & $6114.3 \pm 1767.0$ & 0.240 \\
\hline LSC & $4009.0 \pm 273.1$ & $3554.4 \pm 381.1$ & 0.054 \\
\hline
\end{tabular}

$\tau_{m}$ mean fluorescence lifetime, $p s$ picosecond, SSC short spectral channel, $L S C$ long spectral channel

${ }^{a}$ Mann-Whitney $U$ test $P<0.05$

significant difference in all fluorescence lifetime parameters between the Alzheimer and normal control groups.
However, the fluorescence lifetime is known to be affected by the crystalline lens status. For this reason, the fluorescence lifetime of subjects in this study were compared between pseudophakic and phakic eyes. The average of $\tau_{\mathrm{m}}$ of all pseudophakic eyes, including Alzheimer and normal control groups, was $363 \pm 28$ ps in SSC and $423 \pm 58$ ps in LSC, and $\tau_{\mathrm{m}}$ of phakic subjects was $516 \pm 96$ ps and $412 \pm$ $36 \mathrm{ps}$ in the SSC and LSC, respectively. As expected, $\tau_{\mathrm{m}}$ showed a statistically significant difference in SSC between pseudophakic and phakic eyes $(P=0.001)$. Therefore, comparison between two groups was re-analyzed after dividing eyes into pseudophakic and phakic cohorts. In the phakic cohort, the average $\tau_{\mathrm{m}}$ was significantly increased in the Alzheimer group $(593.9 \pm 93.3 \mathrm{ps}, 454.4 \pm 38.6 \mathrm{ps})$ compared with the normal control group $(475.0 \pm 71.6 \mathrm{ps}$, $394.1 \pm 28.2 \mathrm{ps)}$ in both the SSC and LSC, respectively, $P$ $=0.036$ and 0.024 , Table 2). Of note, there was no statistically significant difference in $\tau_{\mathrm{m}}$ between the two groups when considering pseudophakic eyes alone (Table 2). In the phakic cohort, the $\tau_{1}$ component in the SSC and the $\tau_{2}$ component in the LSC also showed a statistically significant difference between the Alzheimer group and control group $(P=0.008$ and 0.014 , respectively, Table 2$)$

\section{Correlation of fluorescence lifetime with clinical data}

$\mathrm{A} \beta$ and total tau in the CSF and GCIPL thickness on OCT showed significant differences between Alzheimer's and control subjects. These parameters were correlated with FLIO parameters in all subjects, and then separately for the pseudophakic and phakic subgroups.

For the whole cohort, the GCIPL thickness showed a significant negative correlation with $\tau_{\mathrm{m}}$ in LSC $(r=-0.705$, $p<0.001$ with $\tau_{\mathrm{m}}$ Table 3). In pseudophakic eyes alone, several FLIO parameters were significantly correlated with GCIPL thickness. $\tau_{\mathrm{m}}$ in LSC and SSC showed a significant negative correlation with GCIPL thickness $(r=-0.893$ to $-0.795, p<0.05$ for all, Table 3, Fig. 2 a). No other clinical data correlated with FLIO measurements in this subgroup. In phakic eyes, however, $A \beta$ and total tau in CSF were also correlated with FLIO parameters. A $\beta$ and GCIPL thickness was negatively correlated with $\tau_{\mathrm{m}}$, and total tau was positively correlated with $\tau_{\mathrm{m}}$ in these phakic eyes (Table 3 , Fig. 2b-d).

\section{Discussion}

In this pilot study, we observed a correlation between FLIOderived parameters and other clinical and OCT data in patients with suspected early Alzheimer's disease. The diagnosis of Alzheimer's disease is only definitely 
Table 3 Correlation with FLIO parameters and clinical data

\begin{tabular}{|c|c|c|c|c|c|c|c|c|}
\hline & \multicolumn{2}{|l|}{$\tau_{\mathrm{m}}$} & \multicolumn{2}{|l|}{$\tau_{1}$} & \multicolumn{2}{|l|}{$\tau_{2}$} & \multicolumn{2}{|l|}{$\tau_{3}$} \\
\hline & $\mathrm{SSC}$ & LSC & SSC & LSC & SSC & LSC & SSC & LSC \\
\hline \multicolumn{9}{|c|}{ All subjects } \\
\hline \multicolumn{9}{|c|}{$\beta$-Amyloid } \\
\hline$r$ & 0.022 & -0.275 & 0.014 & 0.108 & 0.131 & -0.021 & 0.114 & 0.055 \\
\hline$P$ & 0.921 & 0.193 & 0.948 & 0.616 & 0.541 & 0.924 & 0.595 & 0.799 \\
\hline \multicolumn{9}{|c|}{ Total tau } \\
\hline$r$ & -0.205 & 0.360 & -0135 & -0.151 & -0.332 & -0.057 & -0.349 & -0.302 \\
\hline$P$ & 0.337 & 0.084 & 0.530 & 0.482 & 0.113 & 0.792 & 0.094 & 0.151 \\
\hline \multicolumn{9}{|c|}{ GCIPL thickness } \\
\hline$r$ & 0.021 & -0.705 & -0.198 & 0.180 & 0.177 & -0.396 & 0.218 & 0.029 \\
\hline$P$ & 0.919 & $0.000^{\mathrm{a}}$ & 0.322 & 0.370 & 0.378 & $0.041^{\mathrm{a}}$ & 0.274 & 0.884 \\
\hline \multicolumn{9}{|c|}{ Pseudophakic subjects } \\
\hline \multicolumn{9}{|c|}{$\beta$-Amyloid } \\
\hline$r$ & 0.502 & 0.284 & 0.642 & 0.392 & -0.357 & 0.442 & -0.500 & -0.193 \\
\hline$P$ & 0.204 & 0.496 & 0.086 & 0.337 & 0.385 & 0.272 & 0.207 & 0.646 \\
\hline \multicolumn{9}{|c|}{ Total tau } \\
\hline$r$ & 0.065 & 0.414 & 0.185 & 0.448 & -0.660 & 0.165 & -0.513 & -0.515 \\
\hline$P$ & 0.879 & 0.308 & 0.660 & 0.265 & 0.075 & 0.696 & 0.194 & 0.191 \\
\hline \multicolumn{9}{|c|}{ GCIPL thickness } \\
\hline$r$ & -0.795 & $-\mathbf{0 . 8 8 2}$ & -0.855 & $-\mathbf{0 . 8 9 3}$ & -0.262 & $-\mathbf{0 . 8 5 0}$ & 0.027 & -0.840 \\
\hline$P$ & $0.003^{\mathrm{a}}$ & $0.000^{\mathrm{a}}$ & $0.001^{\mathrm{a}}$ & $0.000^{\mathrm{a}}$ & 0.437 & $0.001^{\mathrm{a}}$ & 0.938 & $0.001^{\mathrm{a}}$ \\
\hline \multicolumn{9}{|c|}{ Phakic subjects } \\
\hline \multicolumn{9}{|c|}{$\beta$-Amyloid } \\
\hline$r$ & -0.387 & -0.577 & -0.516 & 0.089 & -0.130 & -0.594 & -0.150 & -0.308 \\
\hline$P$ & 0.139 & $0.019^{\mathrm{a}}$ & $0.041^{\mathrm{a}}$ & 0.742 & 0.632 & $0.015^{\mathrm{a}}$ & 0.578 & 0.246 \\
\hline \multicolumn{9}{|c|}{ Total tau } \\
\hline$r$ & 0.432 & 0.562 & 0.580 & -0.146 & 0.316 & 0.483 & 0.264 & 0.385 \\
\hline$P$ & 0.095 & $0.024^{\mathrm{a}}$ & $0.019^{\mathrm{a}}$ & 0.590 & 0.233 & 0.058 & 0.323 & 0.141 \\
\hline \multicolumn{9}{|c|}{ GCIPL thickness } \\
\hline$r$ & -0.411 & -0.611 & -0.605 & 0.254 & -0.209 & -0.502 & -0.164 & -0.326 \\
\hline$P$ & 0.114 & $0.012^{\mathrm{a}}$ & $0.013^{\mathrm{a}}$ & 0.342 & 0.437 & $0.048^{\mathrm{a}}$ & 0.544 & 0.218 \\
\hline
\end{tabular}

$\tau_{m}$ mean fluorescence lifetime, SSC short spectral channel, $L S C$ long spectral channel, GCIPL ganglion cell layer + inner plexiform layer

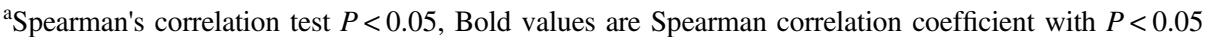

established post mortem through an autopsy of the brain. At present, $A \beta$ and tau protein in CSF, fluorodeoxyglucose and Pittsburg compound B-positron emission tomography in the brain are useful for early diagnosis [20]. However, these methods are expensive, and/or invasive, and some of them require repeat exposure to radiation. The retina is a CNS tissue and shares many similar structural and functional features with the brain. Through the utilization of noninvasive imaging, the retina may be able to provide insight into pathological changes in the brain. Decreased retinal venous blood flow, RGC degeneration in the peripheral retina, and $A \beta$ deposition in the retina have been reported [6-8]. Although the $A \beta$ plaque is the hallmark pathology of
$\mathrm{AD}, \mathrm{A} \beta$ deposition in retina is hard to visualize without a contrast agent such as curcumin [8].

FLIO is a new technique for detecting fluorophores based on their lifetime properties. The retina has several kinds of endogenous fluorophores, including lipofuscin, advanced glycation end products, collagen, and elastin [10, 21, 22] with each fluorophore possessing a characteristic fluorescence lifetime influenced by its environment. The detection of subtle changes and discrimination of these fluorophores might be helpful in detecting functional alterations in retinal diseases.

In our study, we evaluated different stages of Alzheimer's disease using fluorescence lifetime measurements and Alzheimer-related clinical data. Statistically significant 

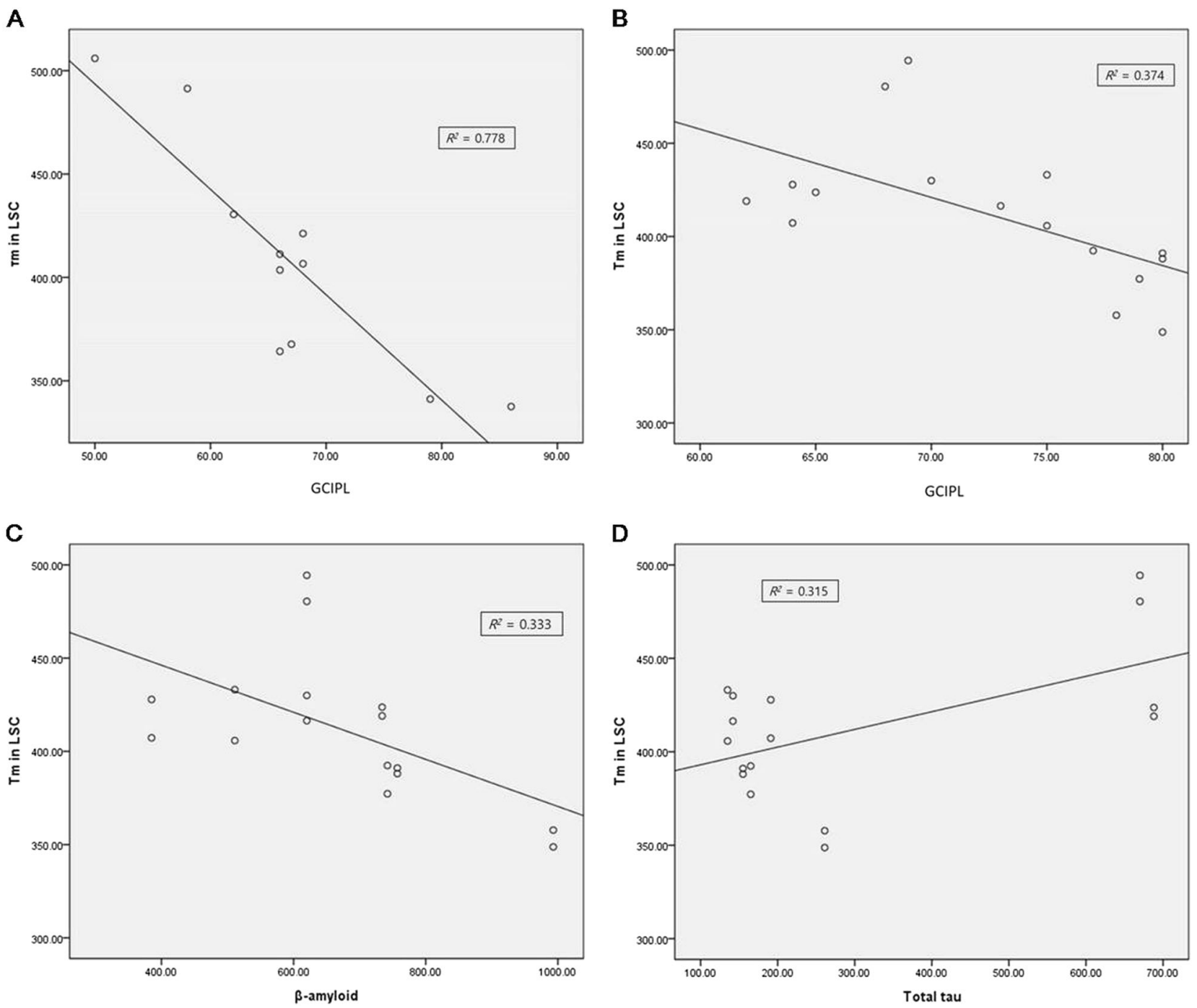

Fig. 2 Correlation plot of mean fluorescence lifetime $\left(\tau_{m}\right)$ with clinical data in phakic and pseudophakic subgroups. a Correlation plot of $\tau_{\mathrm{m}}$ from the LSC with the GCIPL thickness in the pseudophakic group. b Correlation plot of $\tau_{\mathrm{m}}$ from the LSC with the GCIPL thickness in the phakic group. c Correlation plot of $\tau_{\mathrm{m}}$ from the LSC with CSF A $\beta$ level

differences in fluorescence lifetime parameters were found between the Alzheimer and normal control groups, especially among phakic subjects (Table 2 ). In correlation analysis, $\mathrm{A} \beta$ and total tau protein in the CSF and GCIPL thickness on OCT were correlated with FLIO parameters (Table 3). Both $\mathrm{A} \beta$ and tau protein are established biomarkers of AD. The time course of $A \beta$ in the CSF is complex, while $\mathrm{A} \beta$ accumulates and aggregate in the CNS tissue, the level of $\mathrm{A} \beta$ in the CSF decreases before clinical symptoms finally appear. A previous report focused on advanced $\mathrm{AD}$ patients showed a significant correlation between FLIO parameters and tau protein levels [14]. In our study, both tau protein and $\mathrm{A} \beta$, both of which may be considered early biomarkers of Alzheimer's, showed significant correlations. These findings are promising for the

in the phakic group. $\mathbf{d}$ Correlation plot of $\tau_{\mathrm{m}}$ from the LSC with total tau level in CSF of phakic group. LSC $=$ long spectral channel. $\mathrm{SSC}=$ short spectral channel. GCIPL $=$ ganglion cell layer + inner plexiform layer. $\mathrm{CSF}=$ cerebrospinal fluid

possibility of FLIO to identify early AD, but clearly larger longitudinal studies are required.

The fluorescence of the crystalline lens is characterized by a relatively long lifetime, especially in the shortwavelength channel, which is known to result in significant differences of $\tau_{\mathrm{m}}$ between pseudophakic and phakic subjects [13]. Because of this influence of the crystalline lens on the fluorescence lifetime, we followed the convention of previous studies in analyzing pseudophakic and phakic groups separately. We had expected that pseudophakic subjects would be more likely to demonstrate significant correlations with other AD clinical data, as it would be less confounded by the effects of the crystalline lens. Pseudophakic eyes in fact did show the strongest correlation between FLIO parameters and OCT-derived GCIPL 
thickness data. Phakic eyes, however, were the ones that showed the correlation between the FLIO parameters and other clinical data. We have hypothesized that the small sample could be one reason why additional correlations were not observed in the pseudophakic group. A larger longitudinal study would be necessary to elucidate this issue further.

It should be noted, regardless of lens status, more parameters in the LSC (than the SSC) showed a correlation with Alzheimer-related clinical data in our study, consistent with prior reports. In our study, the clinical and OCT data showed a stronger correlation with FLIO parameters for the LSC than the SSC ( $\tau_{\mathrm{m}}$ of LSC with phakic eyes, all FLIO parameters for the LSC and $\tau_{\mathrm{m}}$, for the SSC with pseudophakic eyes). Mean fluorescence lifetime values are known to be significantly dependent on the lens status in the SSC, but independent of the lens status in the LSC [23]. Therefore, our finding that FLIO parameters in the LSC correlated with Alzheimer-associated clinical data suggest that the FLIO parameters, particularly in the LSC, might best reflect the retinal alterations that may be present in Alzheimer's disease.

With respect to OCT, the average thickness of the GCIPL on OCT negatively correlated with the fluorescence lifetime of both channels in pseudophakic subjects and the LSC in phakic channel. Several studies have reported the reduction of RNFL thickness and GCL thickness in AD patients [5, 24, 25]. Since the retinal capillary circulation often mirrors alterations in inner retinal thickness, we expected we might observe OCTA vessel density alterations in our cohort. Bulut et al. [15], for example, have previously reported that the retinal vessel density was significantly lower and the foveal avascular zone was enlarged in $\mathrm{AD}$ patients [15]. Despite the GCIPL correlation, we did not find a correlation between VD and FLIO parameters. Compared to previous studies, however, our study population was different as we only focused on early AD. The lack of VD changes despite GCIPL changes would seem to suggest that microvascular changes may be secondary or follow retinal structural alterations-larger longitudinal studies will again be necessary to confirm this hypothesis.

Our study has significant limitations, including most notably the small cohort size, particularly when subdividing the cohort into phakic and pseudophakic subgroups, which is because many subjects were excluded with strict criteria for accurate result. With this limitation, our study still provided consistent data compared to previous FLIO report of Alzheimer's disease and proved the potential of FLIO for the early diagnosis of Alzheimer's disease. On the other hand, a major strength of our study is the inclusion of $A D$ subjects and controls who had been extensively characterized with CSF, cognitive, and OCT markers.
In summary, FLIO-derived parameters appear to correlate with $A \beta$ and tau levels in the CSF and GCIPL thickness in preclinical AD subjects. Furthermore, FLIO parameters in the LSC showed a stronger correlation than in the SSC. The GCIPL thickness correlated with fluorescence lifetime measurements regardless of the lens status, but $\mathrm{A} \beta$ and tau levels correlated with fluorescence lifetime only in phakic eyes. If these findings can be validated in future longitudinal studies, FLIO could prove to be useful as a non-invasive diagnostic tool for early or subclinical Alzheimer's disease.

\section{Summary}

\section{What was known before}

- FLIO is novel retinal imaging technique be useful for early diagnosis of various retinal disease.

\section{What this study adds}

- FLIO could be useful as a non-invasive diagnostic tool for early or subclinical Alzheimer's disease.

Acknowledgements We thank Yoshihiko Katayama, $\mathrm{PhD}$, and Ali Tafreshi, BS, from Heidelberg Engineering GmBH, Heidelberg, Germany for their valuable advices and providing technical assistance for the FLIO device.

\section{Compliance with ethical standards}

Conflict of interest SRS is a consultant for and receives research support from Optos and Carl Zeiss Meditec, and serves as a consultant for Centervue, and has access to research instruments provided by Heidelberg Engineering, Topcon Medical Systems, Optos, Carl Zeiss Meditec, Nidek, and Centervue. The funders had no role in study design, data collection and analysis, decision to publish, or preparation of the manuscript. The other authors declare that they have no conflict of interest.

Publisher's note: Springer Nature remains neutral with regard to jurisdictional claims in published maps and institutional affiliations.

\section{References}

1. Prince PM, Ali G, Ali G. World Alzheimer Report 2015. The global impact of dementia. London: Alzheimers Disease International; 2015.

2. McKhann GM, Knopman DS, Chertkow H, Hyman BT, Jack CR Jr, Kawas CH, et al. The diagnosis of dementia due to Alzheimer's disease: recommendations from the National Institute on AgingAlzheimer's Association workgroups on diagnostic guidelines for Alzheimer's disease. Alzheimers Dement. 2011;7:263-9.

3. Villemagne VL, Burnham S, Bourgeat P, Brown B, Ellis KA, Salvado $\mathrm{O}$, et al. Amyloid $\beta$ deposition, neurodegeneration and 
cognitive decline in sporadic Alzheimer's disease: a prospective cohort study. Lancet Neurol. 2013;12:357-67.

4. Lim YY, Maruff P, Getter C, Snyder PJ. Discloure of PET amyloid imaging results: a preliminary study of safety and tolerability. Alzheimers Dement. 2016;12:454-8.

5. Hinton DR, Sadun AA, Blanks JC, Miller CA. Optic nerve degeneration in Alzheimer's disease. $\mathrm{N}$ Eng $\mathrm{J}$ Med. 1986;315:485-7.

6. Feke GT, Hyman BT, Stern RA, Pasquale LR. Retinal blood flow in mild cognitive impairment and Alzheimer's disease. Alzheimers Dement. 2015;1:144-51.

7. Bambo MP, Garcia-Martin E, Pinilla J, Herrero R, Satue M, Otin $\mathrm{S}$, et al. Detection of retinal nerve fiber layer degeneration in patients with Alzheimer's disease using optical coherence tomography: searching new biomarkers. Acta Ophthalmol. 2014;92:581-2.

8. Koronyo-Hamaoui M, Koronyo Y, Ljubimov AV, Miller CA, Ko $\mathrm{MK}$, Black KL, et al. Identification of amyloid plaques in retinas from Alzheimer's patients and noninvasive in vivo optical imaging of retinal plaques in a mouse model. Neuroimage. 2011;54: S204-17.

9. Schweitzer D, Hammer M, Schwietzer F, Schweitzer F, Jentsch S, Birckner E, et al. In vivo measurement of time-resolved autofluorescence at the human fundus. J Biomed Opt. 2004;9:121422.

10. Schweitzer D, Schenke S, Hammer M, Schweitzer F, Jentsch S, Birckner E, et al. Towards metabolic mapping of the human retina. Microsc Res Technol. 2007;70:410-9.

11. Dysli C, Quellec G, Abegg M, Menke MN, Wolf-Schnurrbusch U, Kowal J, et al. Quantitative analysis of fluorescence lifetime measurements of the macula using the fluorescence liftime imaging ophthalmoscope in healthy subjects. Invest Ophthalmol Vis Sci. 2014;55:2106-13.

12. Dysli C, Wolf S, Berezin MY, Sauer L, Hammer M, Zinkernagel MS. Fluorescence lifetime imaging ophthalmoscopy. Prog Retin Eye Res. 2017;60:120-43.

13. Schweitzer D, Deutsch L, Klemm M, Jentsch S, Hammer M, Peters S, et al. Fluorescence lifetime imaging ophthalmoscopy in type 2 diabetic patients who have no signs of diabetic retinopathy. J Biomed Opt. 2015;20:61106.

14. Jentsch S, Schweitzer D, Schmidtke KU, Peters S, Dawczynski J, Bär KJ, et al. Retinal fluorescence lifetime imaging ophthalmoscopy measures depend on the severity of Alzheimer's disease. Acta Ophthalmol. 2015;93:e241-7.
15. Bulut M, Kurtuluş F, Gözkaya O, Erol MK, Cengiz A, Akıdan M, et al. Evaluation of optical coherence tomography angiographic findings in Alzheimer's type dementia. $\mathrm{Br} \mathrm{J}$ Ophthalmol. 2018;102:233-7.

16. McGrory S, Taylor AM, Kirin M, Corley J, Pattie A, Cox SR, et al. Retinal microvascular network geometry and cognitive abilities in community-dwelling older people: The Lothian Birth Cohort 1936 study. Br J Ophthalmol. 2017;101:993-8.

17. Golzan SM, Goozee K, Georgevsky D, Avolio A, Chatterjee P, Shen K, et al. Retinal vascular and structural changes are associated with amyloid burden in the elderly: ophthalmic biomarkers of preclinical Alzheimer's disease. Alzheimers Res Ther. 2017;9:13.

18. Kim AY, Chu Z, Shahidzadeh A, Wang RK, Puliafito CA, Kashani AH. Quantifying microvascular density and morphology in diabetic retinopathy using spectral-domain optical coherence tomography angiography. Invest Ophthalmol Vis Sci. 2016;57:362-70.

19. Uji A, Balasubramanian S, Lei J, Baghdasaryan E, Al-Sheikh M, Sadda SR. Impact of multiple en face image averaging on quantitative assessment from optical coherence tomography angiography images. Ophthalmology. 2017;124:944-52.

20. Rabinovici GD, Rosen HJ, Alkalay A, Kornak J, Furst AJ, Agarwal N, et al. Amyloid vs FDG-PET in the differential diagnosis of AD and FTLD. Neurology. 2011;77:2034-42.

21. Delori FC, Dorey KC, Staurenghi G, Arend O, Goger DG, Weiter JJ. In vivo fluorescence of the ocular fundus exhibits retinal pigment epithelium lipofusin characteristics. Invest Ophthalmol Vis Sci. 1995;36:718-29.

22. Ishibashi T, Murata T, Hanagai M, Nagai R, Horiuchi S, Lopez $\mathrm{PF}$, et al. Advanced glycation end products in age related macular degeneration. Arch Ophthalmol. 1998;116:1629-32.

23. Dysli C, Wolf S, Zinkernagel MS. autofluorescence lifetimes in geographic atrophy in patients with age-related macular degeneration. Invest Ophthalmol Vis Sci. 2016;57:2479-87.

24. Marziani E, Pomati S, Ramolfo P, Cigada M, Giani A, Mariani C, et al. Evaluation of retinal nerve fiber layer and ganglion cell layer thickness in Alzheimer's disease using spectral-domain optical coherence tomography. Invest Ophthalmol Vis Sci. 2013;54:5953-8.

25. Lu Y, Li Z, Zhang X, Ming B, Jia J, Wang R, et al. Retinal nerve fiber layer structure abnormalities in early Alzheimer's disease: evidence in optical coherence tomography. Neurosci Lett. 2010;480:69-72. 